\title{
OPEN Gray matter declines with age and hearing loss, but is partially maintained in tinnitus
}

\begin{abstract}
Elouise A. Koops ${ }^{1,2,3 凶}$, Emile de Kleine ${ }^{1,2} \&$ Pim van Dijk ${ }^{1,2}$
The impact of age-related hearing loss extends beyond the auditory pathway and impacts brain areas related to cognitive impairment and even dementia. The presence of tinnitus, a sensation of sound that frequently co-occurs with hearing loss, is additionally linked to cognitive decline. Interestingly, structural neuroimaging studies have reported that hearing loss may precede or modulate the onset of cognitive impairment. In this study, we aimed to disentangle the effects of age, hearing loss, and tinnitus on gray matter structure. In total, 39 participants with hearing loss and tinnitus, 21 with hearing loss but without tinnitus, and 39 controls were included in this voxel- and surfacebased morphometry MRI study. Whole brain volume and surface thickness measures were compared between the groups. Age-related gray matter volume decline was observed in all groups. Several brain areas showed smaller gray matter volume and cortical surface thickness in hearing loss without tinnitus, relative to controls. This reduction was observed both within and outside of the auditory pathway. Interestingly, these reductions were not observed in participants with tinnitus, who had similar hearing loss and were of similar age. Since we have tools to improve hearing loss, hearing screening may aid in the battle against cognitive decline.
\end{abstract}

Hearing loss is the most prevalent acquired sensory impairment and it affects a large part of the ageing population worldwide ${ }^{1}$. Hearing loss not only has an impact on the quality of hearing, it is also associated with an increased likelihood of cognitive impairments and even dementia. Presently, with a globally ageing population, the prevalence of hearing loss is rising ${ }^{2}$ together with the interest in the impact of acquired and age-related hearing loss on the brain. In light of this, it has become clear that sensory impairments are not restricted to the peripheral sensory organs, i.e. the ear, and changes to the brain are involved $\operatorname{too}^{3-6}$. Correspondingly, hearing loss related brain changes appear to affect other faculties than hearing. Several studies have reported a relation between age-related hearing loss, or presbycusis, and poorer cognitive functioning and even dementia ${ }^{7-13}$. For instance, longitudinal data showed that hearing impairment is associated with a larger likelihood of developing cognitive impairment ${ }^{7}$. Furthermore, a recent study identified hearing loss as an independent modifiable risk factor for cognitive decline ${ }^{10}$. For a comprehensive overview, see Jafari et al., $2019^{14}$. It thus appears that hearing loss and cognitive decline do not only appear in the same population, hearing loss may precede or modulate the onset of cognitive impairment. Therefore, the effect of hearing loss on the brain is a potential window to understand cortical changes related to ageing.

Tinnitus is a symptom that often co-occurs with hearing loss, and is characterized by the perception of sound in the absence of an external acoustic stimulus. Since there is a strong link between hearing loss and neural plasticity and a strong association between hearing loss and tinnitus, it has been hypothesized that tinnitus is linked to neuroplasticity as well ${ }^{15-20}$. Furthermore, similar to hearing loss, tinnitus has been linked to an increased risk of cognitive deficits ${ }^{21}$. Several studies indicate that the presence of tinnitus has a negative impact on memory, executive functioning, and quality of life ${ }^{22}$. Interestingly, one study noted that the extent of the cognitive deficits is linked to the perceived severity of the tinnitus ${ }^{21}$. Thus, in addition to the strong link between tinnitus and hearing loss, it has been reported that tinnitus by itself has a negative impact on cognitive functioning. Therefore, the factor of tinnitus has to be considered when investigating cortical changes in a population with hearing loss.

To date, neuro-imaging studies identified several changes in gray-matter brain volume related to presbycusis (i.e. age-related hearing loss), either with Voxel-Based-Morphometry (VBM), Surface-Based-Morphometry

\footnotetext{
${ }^{1}$ Department of Otorhinolaryngology/Head and Neck Surgery, University Medical Center Groningen, University of Groningen, P.O. Box 30.001, 9700 RB Groningen, The Netherlands. ${ }^{2}$ Graduate School of Medical Sciences (Research School of Behavioural and Cognitive Neurosciences), University of Groningen, Groningen, The Netherlands. ${ }^{3}$ Cognitive Neuroscience Center Groningen, University of Groningen, Groningen, The Netherlands. ${ }^{\boxplus}$ email: e.a.koops@umcg.nl
} 


\begin{tabular}{|l|l|l|l|}
\hline Group & Mean age (years) & Standard deviation & N \\
\hline Control & 45.7 & 13.5 & 39 \\
\hline Hearing loss & 62.6 & 8.8 & 21 \\
\hline Tinnitus + hearing loss & 59.2 & 9.3 & 39 \\
\hline
\end{tabular}

Table 1. Demographics of participants. The hearing loss groups, with and without tinnitus, did not differ with respect to age $(\mathrm{p}=0.419)$. The control group was significantly younger than both hearing loss groups, with and without tinnitus $(\mathrm{p}<0.0001)$.

(SBM) or other types of automated morphometry. The reported affected areas extend beyond the auditory pathway and correspond to areas related to cognitive impairment and dementia. In both unilateral and bilateral hearing loss, the decrease in brain volume in the auditory cortex, anterior cingulate, and prefrontal cortex correlated with the loss in hearing sensitivity ${ }^{23-27}$. Furthermore, VBM studies that investigated cognitive decline and presbycusis reported accelerated cortical volume decline in the presence of presbycusis ${ }^{25,26,28,29}$. In line with this, the results of SBM studies indicated a relationship between cognitive impairment and presbycusis. These surface-based studies identified gray matter atrophy in the primary auditory cortex, the cingulate cortex, the precuneus, the insula, and the parietal cortex in the presence of presbycusis ${ }^{29,30}$. Surprisingly, one study reported an association between hearing loss and an increase in the gray matter of the right angular gyrus ${ }^{31}$. Nevertheless, pervasive decreases in gray matter volume and surface thickness, which are generally associated with cognitive decline, may additionally be linked to age-related hearing loss.

Furthermore, recent neuroimaging studies have tried to identify the central correlate of tinnitus. These studies identified changes in gray matter volume in auditory, prefrontal, and limbic areas that were associated with the presence of tinnitus or its characteristics. More specifically, reductions in gray matter were reported for the prefrontal cortex ${ }^{32-37}$, the subcallosal area ${ }^{35,38}$, precuneus ${ }^{32}$, supra marginal gyrus ${ }^{33}$, orbitofrontal cortices ${ }^{39}$, cingulate $^{36}$, insular cortex ${ }^{36}$, and the pre- and postcentral gyrus ${ }^{36}$. However, the manifestation of this effect in the temporal and thalamic areas is variable in these previously published studies. For the temporal area, it has been reported that an increase in auditory cortex gray matter related to the presence of tinnitus ${ }^{37,39}$, whereas tinnitus distress related to a decrease in auditory cortex gray matter ${ }^{36}$. Similarly, for the thalamus both increases and decreases in volume were reported ${ }^{36,38}$. Overall, the reported changes in cortical structure that are associated with tinnitus vary between studies, and are in some instances contradictory. The heterogeneity of the tinnitus population, the confound of hearing loss, differences in age as well as non-corresponding techniques and lenient statistical thresholds have been named as contributing factors to this discrepancy ${ }^{32,40}$.

Since emerging evidence indicates that acquired hearing loss and tinnitus are associated with age-related cognitive impairment, the identification of differences in brain structure that are specific to each of these conditions may help us to develop appropriate treatments that not only aid hearing or improve tinnitus, but may additionally prevent cognitive decline. A current challenge is to determine if the structural characteristics that might contribute to cognitive impairment should be attributed to hearing loss, to tinnitus, or related factors such as age. Hence, the aim of this study was to disentangle the effects of age, acquired hearing loss, and tinnitus on the gray matter of the brain.

\section{Results}

The data of 89 participants are presented, of which 39 had tinnitus and hearing loss (THL group), 21 participants had hearing loss but no tinnitus (HL group), and 39 controls with neither tinnitus and no or minimal hearing loss (CO group). Table 1 contains the demographics of the three participant groups. The mean age of both hearing loss groups differed significantly from the control group whereas the hearing loss groups did not differ from each other. The hearing loss groups, with and without tinnitus, do not differ significantly on any of the frequencies (corrected for multiple comparisons; see description Fig. 1). However, the hearing thresholds of both hearing loss groups differed significantly from those of the control group on all frequencies. Age and average hearing loss (HF-PTA) showed a significant positive correlation across all participants $(r=0.6125, p<0.0001$; Fig. 2). Analysis run on group level showed that only in the control group there was a significant correlation between average hearing loss and age (THL: $\mathrm{R}=0.20, \mathrm{p}=0.22 ; \mathrm{HL}: \mathrm{R}=0.10, \mathrm{p}=0.66 ; \mathrm{CO}: \mathrm{R}=0.76, \mathrm{p}<0.0001$ ).

Morphometry. Voxel based morphometry. A voxel-based multiple regression model was used to explain differences in gray matter volume in terms of age, sex, the presence of tinnitus, and average hearing loss (HFPTA). In addition, Total Intracranial Volume (TIV) was included as a covariate. This analysis demonstrated that age was a significant predictor for gray matter volume in our study sample (family-wise error $(\mathrm{FWE})<0.05$, cluster size $\mathrm{k}>20$; see Table 2). Specifically, age was inversely related to gray matter volume in the cingulate and auditory cortical areas (see Fig. 3). None of the other predictors were significantly associated with gray matter volume. However, non-significant associations were identified $(p<0.001$ uncorrected, $k>20)$; The presence of tinnitus was associated with greater gray matter volume in the bilateral lingual gyrus, bilateral crus of the cerebellum, left secondary visual cortex, and the left retrosubicular area. High-frequency hearing loss was associated with less gray matter volume in the right cortical auditory areas and the left cerebellar area. Even though these findings were non-significant, they are mentioned here since other studies identified similar areas as related to hearing loss and tinnitus (see Discussion). 


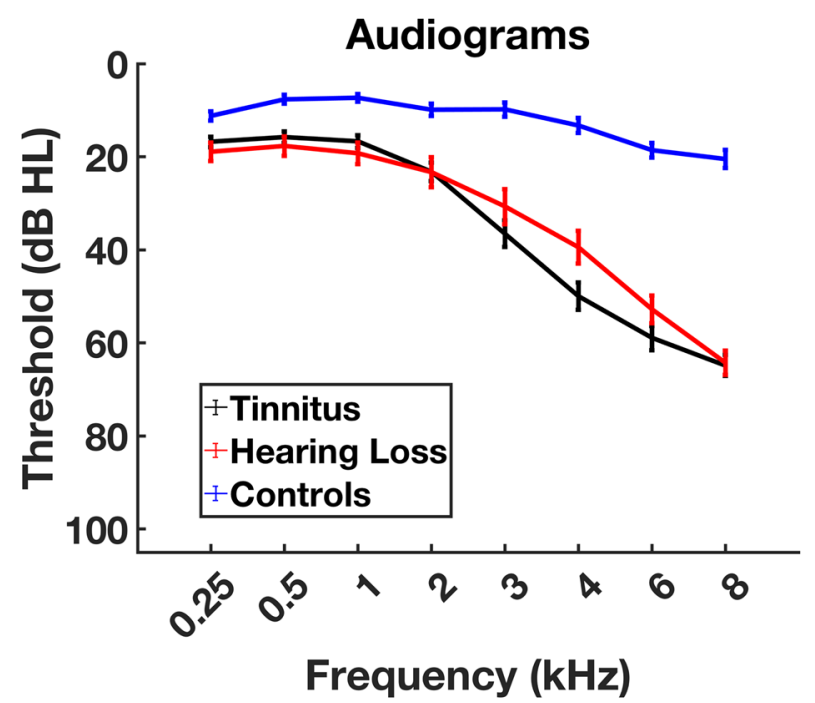

Figure 1. Average hearing thresholds per group with their corresponding standard errors. Pure tone audiometry was used to test octave frequencies from 0.25 to $8 \mathrm{kHz}$, and 3 and $6 \mathrm{kHz}$. The two groups with hearing loss, with and without tinnitus, were not significantly different in terms of age $(\mathrm{p}=.419, \mathrm{t} 1.359)$ and hearing loss $[\mathrm{p}=.209(0.25 \mathrm{kHz}) ; \mathrm{p}=.3(0.5 \mathrm{kHz}) ; \mathrm{p}=.4161(\mathrm{kHz}) ; \mathrm{p}=.683(2 \mathrm{kHz}) ; \mathrm{p}=0.812(3 \mathrm{kHz}) ; 4 \mathrm{kHz}$ $\mathrm{p}=.976(4 \mathrm{kHz}) ; \mathrm{p}=.783(6 \mathrm{kHz}) ; \mathrm{p}=.974(8 \mathrm{kHz})]$.

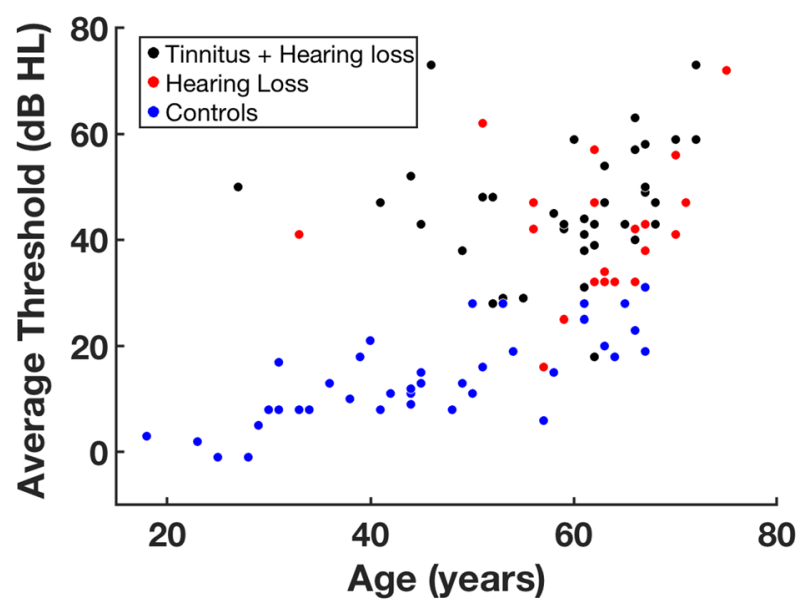

Figure 2. Average hearing threshold of all participants, plotted against age. The control group was significantly younger than both the hearing loss group with $(\mathrm{p}<0.0001, \mathrm{t} 5.1)$ and without tinnitus $(\mathrm{p}<0.0001, \mathrm{t} 5.9)$. The average threshold was computed for the frequencies of 2,4 , and $8 \mathrm{kHz}$.

Pair-wise comparisons between groups. Pair-wise comparisons on volumetric data of the three participant groups were investigated with Threshold Free Cluster Enhanced (TFCE) two-sample t-tests, that were performed on the modulated gray-matter volume. The pairwise group comparisons tested were: tinnitus and hearing loss versus hearing loss (THL vs. HL), hearing loss versus controls (HL vs. CO), and tinnitus and hearing loss versus controls (THL vs. CO). In addition, the inverse of all these comparisons was tested, resulting in a total of six pairwise group comparisons. All of the pair-wise comparisons were controlled for age and TIV.

The first analysis revealed a difference in gray matter volume between the two hearing loss groups, with and without tinnitus (THL vs. HL). This group comparison showed significantly higher gray matter volume in the bilateral Lingual Gyrus (BA 37, FWE $<0.05, \mathrm{k}=363(\mathrm{~L}) / 277(\mathrm{R})$ ) for the tinnitus group; see Fig. 4A and Table 3. In this comparison, there were no areas that showed significantly smaller gray matter volume. Second, in the hearing loss group without tinnitus, there were no areas in which the volume was larger than in the control group (HL vs. CO). However, gray matter volume was smaller in the bilateral middle temporal gyri, left inferior temporal gyrus, right orbitofrontal area, right cingulate cortex, left fusiform gyrus, and right temporopolar area; see Fig. 4B and Table 4. Finally, in the comparison of hearing loss with tinnitus (THL) versus controls (CO), none of the differences reached significance. See Tables 2, 3, and 4 for the coordinates and cluster sizes of the reported VBM differences. 


\begin{tabular}{|c|c|c|c|c|c|}
\hline \multirow[b]{2}{*}{ Area } & \multicolumn{2}{|l|}{ Cluster } & \multicolumn{3}{|c|}{$\begin{array}{l}\text { MNI coordinates } \\
(\mathrm{mm})\end{array}$} \\
\hline & P (FWE-cor) & $\mathbf{k}$ & $\mathbf{X}$ & $\mathbf{Y}$ & $\mathbf{Z}$ \\
\hline Superior temporal gyrus $(\mathbf{R})$ & $<0.0005$ & 4316 & 45 & -10 & -3 \\
\hline Insula & & & 48 & 6 & -8 \\
\hline${ }^{*}$ Cluster extends to include BA $22 \& 41$ & & & 56 & -2 & 0 \\
\hline Superior temporal gyrus $(\mathrm{L})$ & $<0.0005$ & 3125 & -45 & -6 & -10 \\
\hline Insula & & & -46 & -10 & 2 \\
\hline${ }^{\star}$ Cluster extends to include the insula, BA $22 \& 41$ & & & -33 & -21 & 12 \\
\hline Putamen $(\mathrm{L})$ & $<0.0005$ & 382 & -26 & 9 & -12 \\
\hline${ }^{*}$ Cluster extends to include the subcallosal gyrus and amygdala & & & -26 & 18 & 0 \\
\hline Fusiform gyrus $(\mathbf{R})$ & $<0.0005$ & 228 & 24 & -40 & -16 \\
\hline \multicolumn{6}{|c|}{${ }^{*}$ Cluster extends to include the parahippocampal gyrus fusiform gyrus and lingual gyrus } \\
\hline Fusiform gyrus $(\mathrm{L})$ & $<0.0005$ & 428 & -22 & -44 & -14 \\
\hline Parahippocampal gyrus & & & -26 & -28 & -18 \\
\hline Lingual gyrus & & & -18 & -46 & -6 \\
\hline \multirow[t]{3}{*}{ Cingulate gyrus $(\mathrm{L})$} & $<0.0005$ & 1264 & -14 & -44 & 42 \\
\hline & & & 9 & -45 & 33 \\
\hline & & & -10 & -51 & 38 \\
\hline Middle temporal gyrus (R) & $<0.0005$ & 123 & 58 & -57 & -3 \\
\hline Hippocampus (R) & $<0.0005$ & 144 & 22 & -32 & -4 \\
\hline Calcarine sulcus V1 (R) & $<0.0005$ & 158 & 2 & -80 & 6 \\
\hline \multicolumn{6}{|l|}{${ }^{*}$ Cluster extends to include the lingual gyrus } \\
\hline Mamillary bodies & $<0.0005$ & 53 & 0 & -9 & -10 \\
\hline Middle cingulate gyrus (R) & $<0.0005$ & 402 & 3 & 27 & 33 \\
\hline \multirow[t]{2}{*}{ Anterior cingulate } & & & 2 & 39 & 21 \\
\hline & & & 4 & 16 & 40 \\
\hline Posterior cingulate gyrus $(\mathrm{R})$ & $<0.0005$ & 222 & 3 & -63 & 18 \\
\hline Supramarginal gyrus $(\mathbf{R})$ & 0.001 & 34 & 40 & -46 & 56 \\
\hline Angular gyrus (L) & $<0.0005$ & 116 & -46 & -68 & 32 \\
\hline Olfactory area $(\mathbf{R})$ & $<0.0005$ & 104 & 24 & 12 & -14 \\
\hline Putamen (R) & 0.001 & 31 & 28 & 18 & 2 \\
\hline Middle temporal gyrus $(\mathrm{L})$ & 0.001 & 38 & -63 & -46 & 8 \\
\hline Inferior frontal gyrus $(\mathrm{R})$ & $<0.0005$ & 108 & 44 & 20 & 0 \\
\hline BA $45(\mathrm{R})$ & & & 54 & 18 & 3 \\
\hline \multicolumn{6}{|l|}{${ }^{*}$ Cluster extends to include the insula and orbitofrontal cortex } \\
\hline Superior temporal gyrus $(\mathrm{L})$ & 0.001 & 44 & -56 & -60 & 21 \\
\hline \multirow[t]{2}{*}{ BA 7 (R) } & $<0.0005$ & 67 & 3 & -64 & 45 \\
\hline & & & 6 & -63 & 36 \\
\hline
\end{tabular}

Table 2. The coordinates for the local maxima of areas that show an inverse relation between the gray matter volume and age.

Surface based morphometry. In addition to the volumetric measures of the VBM method, surface-based morphometry (SBM) was performed to measure cortical thickness and gyrification ${ }^{41}$. The cortical thickness was significantly reduced in the hearing loss group without tinnitus compared to the control group. This reduction was observed in the left supramarginal gyrus and angular gyrus, extending posteriorly to the inferior parietal gyrus, and the left lateral occipital, middle temporal, and inferior temporal areas (TFCE, FWE < 0.05; see Fig. 5). No significant differences in cortical thickness were observed between both hearing-impaired groups, with and without tinnitus, and between the hearing loss group with tinnitus and the control group. The gyrification analyses did not show any significant differences between the groups.

Post-hoc analysis. To assess if the auditory cortex is distinctively affected by age in comparison to other primary sensory or motor related areas, post-hoc region-of-interest (ROI) analyses were performed on both the gray matter volume and the surface-based thickness data, inspired by Profant et al. ${ }^{29}$. Three ROI areas were included: the visual cortex (V1), motor cortex (M1), and the auditory cortex (A1). ROI determination was performed using the HCP_MMP1 atlas for the thickness parcellations, and the atlas provided by Neuromorphometric Inc. for the volume parcellations ${ }^{42}$.

The group differences in surface thickness and volume of gray matter that were identified in the three ROIs are depicted in Fig. 6. Left dominant laterality was observed for the auditory cortex, both for the volumetric and 
A

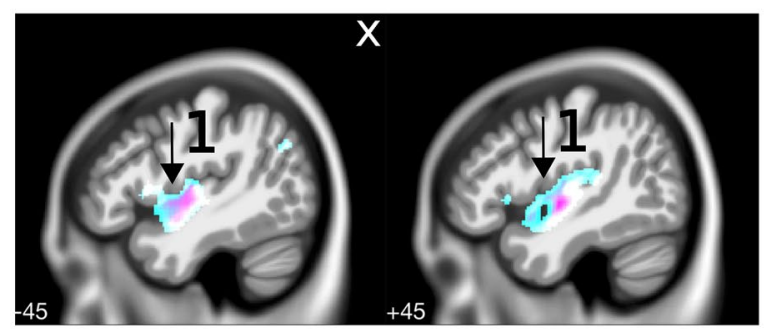

B

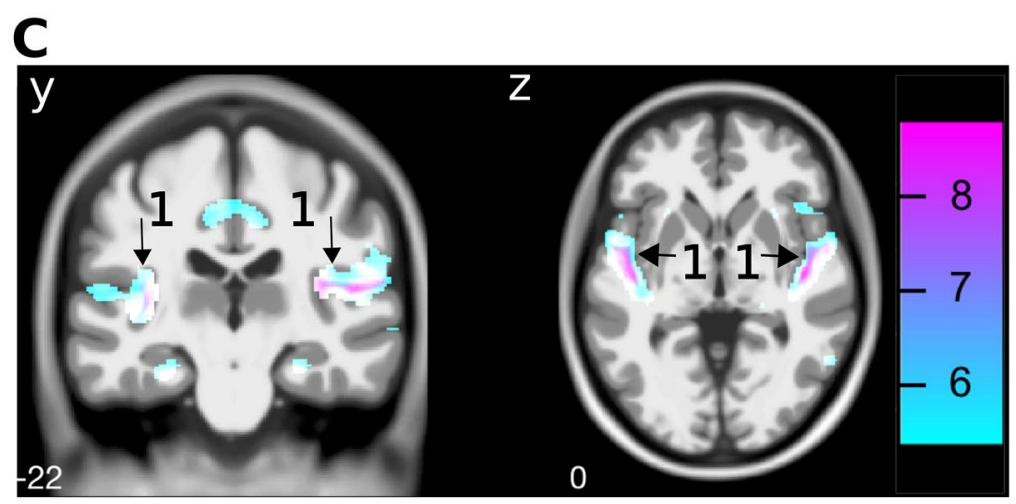

Figure 3. Cortical gray matter volume differences related to age. A multiple regression model with the covariates age, sex, tinnitus, and hearing thresholds at 2,4 , and $8 \mathrm{kHz}$ indicated that increasing age was associated with less gray matter volume in (A) temporal areas (including the bilateral auditory cortices (1)) and (B) anterior (2), middle (3) and posterior (4) cingulate areas, at $\mathrm{FWE}<0.05$. Scale bar indicates $\mathrm{T}$ values.

A

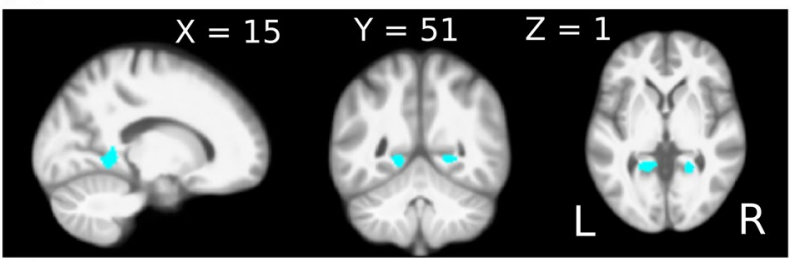

$\overline{\mathbf{B}}$

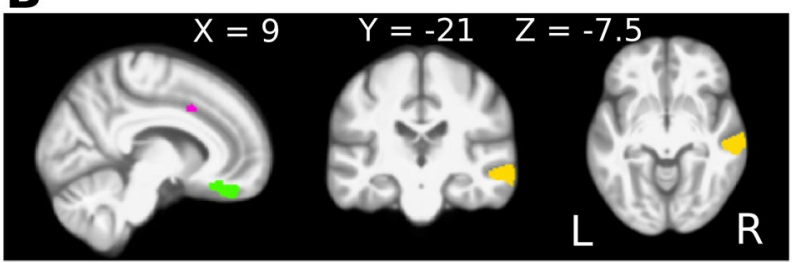

Figure 4. Gray matter volume differences associated with hearing loss and with tinnitus. Threshold free cluster enhancement (TFCE) was employed to investigate group differences in modulated gray matter images. (A) Comparison between the hearing loss groups, with and without tinnitus. In the group with tinnitus, larger gray matter volume of the bilateral lingual gyri was observed. These areas are depicted in blue on a medial view of the sagittal plane of the right hemisphere, and a coronal and axial cross section of the entire brain. (B) Comparison between the hearing loss group without tinnitus and the control group. In the hearing loss group without tinnitus less gray matter volume was observed in the cingulate cortex (pink), the orbitofrontal cortex (green), and the middle temporal gyri (yellow), depicted on a medial view of the sagittal plane of the right hemisphere. Additional clusters with less gray matter volume, not shown here, were observed in the superior and inferior temporal gyri and the fusiform gyrus (see Table 4). The comparison between the hearing loss group with tinnitus and the controls revealed no significant differences and is therefore not shown in this figure. 


\begin{tabular}{|l|l|l|l|l|l|}
\hline Area & Cluster & Peak-cluster & \multicolumn{4}{|l|}{ MNI coordinates $(\mathbf{m m})$} \\
\hline & P (FWE-cor) & K & X & Y & Z \\
\hline Lingual gyrus (L) & 0.019 & 363 & -20 & -46 & -3 \\
\hline Lingual gyrus (R) & 0.031 & 277 & 24 & -46 & -2 \\
\hline & 0.039 & & 18 & -44 & 12 \\
\hline
\end{tabular}

Table 3. The coordinates for the local maxima of gray matter volume differences between the hearing loss groups with and without tinnitus. These areas shows more gray matter volume in the hearing loss group with tinnitus, compared to the hearing loss group without tinnitus. The comparison between the tinnitus group and the controls did not reveal a significant group difference, and is therefore not shown in this table.

\begin{tabular}{|c|c|c|c|c|c|}
\hline \multirow[t]{2}{*}{ Area } & \multirow{2}{*}{\begin{tabular}{|l} 
Cluster \\
P (FWE-cor)
\end{tabular}} & \multirow{2}{*}{\begin{tabular}{|l} 
Peak-cluster \\
$\mathbf{k}$ \\
\end{tabular}} & \multicolumn{3}{|c|}{$\begin{array}{l}\text { MNI Coordinates } \\
(\mathbf{m m})\end{array}$} \\
\hline & & & $\mathbf{x}$ & $\mathbf{Y}$ & $\mathrm{Z}$ \\
\hline Middle temporal gyrus (L) & 0.016 & 340 & -62 & -33 & -16 \\
\hline \multicolumn{6}{|c|}{${ }^{*}$ Cluster extends to include the inferior temporal gyrus } \\
\hline \multirow[t]{2}{*}{ Middle temporal gyrus (R) } & 0.019 & 1144 & 63 & -26 & -4 \\
\hline & 0.019 & & 57 & -26 & -10 \\
\hline${ }^{*}$ Cluster extends to include the insula & 0.020 & & 66 & -15 & -12 \\
\hline \multirow[t]{2}{*}{ Sub-gyral area $(\mathrm{R})$} & 0.022 & 436 & 10 & 39 & -21 \\
\hline & 0.025 & & 9 & 30 & -16 \\
\hline \multicolumn{6}{|c|}{${ }^{*}$ Cluster extends to include the orbitofrontal cortex and amygdala } \\
\hline Middle cingulate gyrus $(\mathrm{R})$ & 0.044 & 44 & 10 & 12 & 39 \\
\hline \multirow[t]{2}{*}{ Fusiform gyrus $(\mathrm{L})$} & 0.045 & 59 & -40 & -48 & -22 \\
\hline & 0.049 & & -34 & -40 & -18 \\
\hline Superior temporal gyrus $(\mathbf{R})$ & 0.046 & 70 & 52 & 2 & -3 \\
\hline \multicolumn{6}{|c|}{${ }^{*}$ Cluster extends to include the temporal pole } \\
\hline Fusiform gyrus (L) & 0.048 & 24 & -52 & -46 & -21 \\
\hline
\end{tabular}

Table 4. The coordinates for the local maxima obtained via a two-sample $t$-test, comparing the hearing loss group without tinnitus to the control group. The areas identified show less gray matter volume in the hearing loss group without tinnitus compared to the controls.

surface-based methods. No group related differences in laterality were observed. Therefore, subsequent analyses were performed on the average of left and right ROIs. First, a multivariate analysis of covariance showed that there was a significant decline in both gray matter volume and surface thickness with age in all three ROIs, see Table 5. Second, the effect of group on gray matter volume was tested for the three ROIs included, adjusted for multiple comparisons in the strictest sense via a Bonferroni correction and corrected for age. This analysis showed that there was no significant effect of group on cortical volume or thickness for V1 and M1 after correcting for age (Table 5, Group). Moreover, A1 gray matter volume was not significantly different between the groups. Nonetheless, both the median cortical thickness and the median gray matter volume of A1 were smallest in the hearing-impaired group without tinnitus, and largest in the controls. However, only the thickness measures were significantly different between the groups (see Table 5 and Fig. 6A, right panel). To conclude, the effect of age on all of the included cortical areas is strong, and the only group related difference was observed in the thickness of the auditory cortex.

\section{Discussion}

The aim of this study was to disentangle the effect of age, hearing loss, and tinnitus on the structure of cortical gray matter. Three groups were included: (1) hearing loss and tinnitus, (2) hearing loss without tinnitus, and (3) controls without hearing loss or tinnitus. The first result is that age was significantly related to gray matter volume decline in all three groups. Second, in the hearing loss group with tinnitus significantly higher gray matter volume was observed in the lingual gyri compared to the hearing loss group without tinnitus. Third, in hearing loss without tinnitus significant gray matter reductions, relative to controls, were observed in the temporal gyri, orbitofrontal area, cingulate cortex, fusiform gyrus, and the temporopolar area. Additionally, the thickness of the cortical surface was significantly less in the supramarginal gyrus, inferior parietal area, lateral occipital area, middle temporal and inferior temporal areas in hearing loss without tinnitus, compared to controls. These reductions were not observed in hearing loss participants with tinnitus, who were of similar age and had similar hearing thresholds. 
$\mathbf{A}$

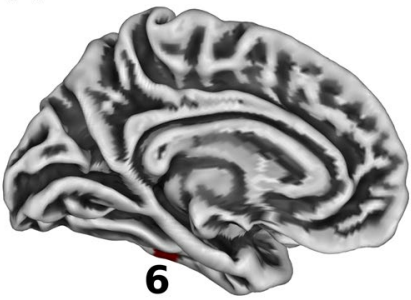

B
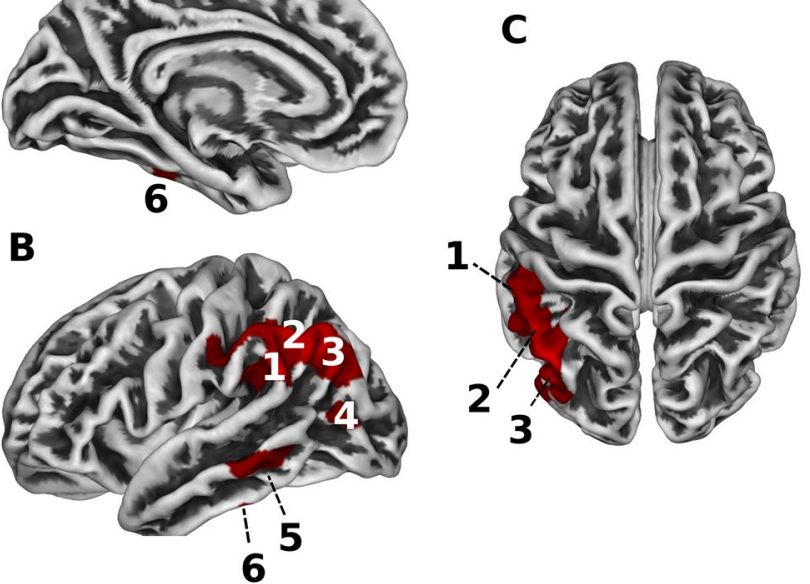

Figure 5. Reduction in cortical surface thickness in the hearing loss group without tinnitus compared to the control group. The three panels show a medial (A) and lateral (B) view of the left hemisphere, and a top view (C) of the entire brain. Areas that contained less cortical surface thickness in the hearing loss group without tinnitus compared to the control group (FWE $<0.05$, TFCE) are shown in red. These areas include the supramarginal gyrus (1), the angular gyrus (2), the inferior parietal lobule (3), lateral occipital area (4), and the middle temporal (5) and inferior temporal areas (6), all on the left side of the brain.

Age related differences. The multivariate analysis of the association between age, hearing loss, and tinnitus showed that age is significantly related to gray matter volume reductions. This effect was observed in the auditory cortices, in the cingulate areas, and in the orbitofrontal area. These specific brain areas have been described in other studies on aging and brain volume $e^{29,43-45}$. Our post-hoc analysis on gray matter volume of the visual, motor, and auditory cortices showed a larger effect of age on the auditory than on the visual and sensorimotor cortex. Note that a part of the participants in our study were hearing impaired, either with or without tinnitus. Overall, age appears to have a more detrimental impact on the auditory cortex than it does on the visual or motor cortices. Moreover, hearing loss appears to selectively aggravate the detrimental effect of age on the gray matter of the auditory cortex.

Hearing loss related differences. In addition to the effect of age, acquired hearing loss has a distinct effect on the gray matter brain volume of the temporal gyri, the orbitofrontal cortex, middle cingulate and fusiform gyrus. Moreover, in the present study hearing loss was associated with differences in the cortical surface thickness of the supramarginal gyrus, angular gyrus, inferior parietal area, occipital and temporal areas. Our results are in agreement with previous reports on reduced gray matter volume in the temporal lobes and cingulate area of individuals with hearing $\operatorname{los}^{23-26,28}$. Similarly, our surface based thickness measure indicated that hearing loss without tinnitus is related to less gray matter thickness in the parietal cortex and insula, which is in line with the reported results of an earlier study ${ }^{30}$. Hearing loss, in addition to age, is thus related to a reduction in the cortical volume and thickness of areas within and beyond the auditory system.

Tinnitus related differences. Our results support the finding of the study by Husain et al..$^{24}$ that structural changes are more pronounced in hearing loss without tinnitus than in hearing loss with tinnitus. In line with this, the hearing loss group without tinnitus in our study had significantly lower gray matter volume and surface thickness in several areas compared to controls, whereas the hearing loss group with tinnitus did not. Likewise, Husain et al. ${ }^{24}$ reported no significant differences in gray matter volume between hearing loss with tinnitus and healthy controls in participants with hearing thresholds similar to our study. Interestingly, this finding suggests that in hearing loss the additional presence of tinnitus is related to a more limited decline in gray matter and thus better preservation of gray matter in several temporal, frontal, and occipital areas.

In the hearing loss group with tinnitus, the gray matter volume of the lingual gyri was larger than in those without tinnitus. Previously, the lingual gyrus has been connected to tinnitus in studies that investigated restingstate connectivity. These studies reported tinnitus related increases in connectivity of the left lingual gyrus with the left auditory cortex ${ }^{46}$ and decreased connectivity of the left lingual gyrus with the auditory resting state network ${ }^{47}$ compared to controls with normal hearing. Studies that compared tinnitus participants to hearing loss matched controls reported increased connectivity of the bilateral lingual gyrus with the attention and short-term memory networks ${ }^{48}$, increased connectivity of the lingual gyri with the default mode network, and a trend towards decreased connectivity with the auditory resting state network ${ }^{47}$. Our results indicate that in the presence of tinnitus the gray matter volume of the lingual gyrus is altered along with the functional connectivity differences reported in literature. To our knowledge there is currently no research published on specific structural 
$\mathbf{A}$

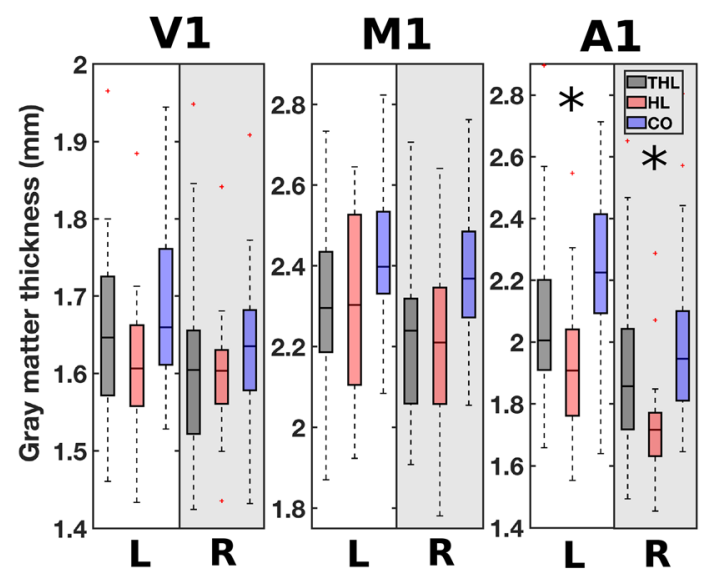

B

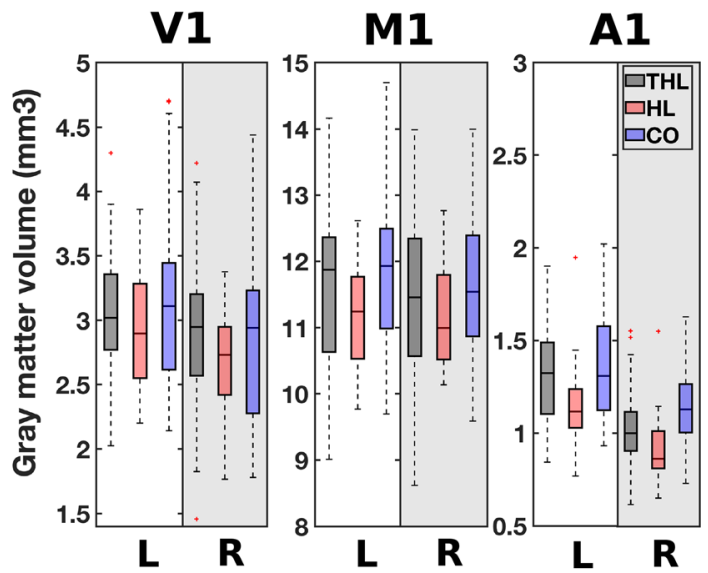

Figure 6. Cortical surface thickness (A) and gray matter volume (B) in primary visual cortex (V1), primary motor cortex (M1) and primary auditory cortex (A1) for the three participant groups: THL (hearing loss with tinnitus); HL (hearing loss without tinnitus); $\mathrm{CO}$ (controls without tinnitus or hearing loss). The boxplots show the cortical surface thickness and the gray matter volume for both the left (L) and right (R) hemispheres. Significance, corrected for multiple comparisons at a stringent Bonferroni level, is indicated with an asterisk.

\begin{tabular}{|l|l|l|l|l|}
\hline \multirow{2}{*}{} & Volume & Thickness \\
\cline { 2 - 5 } & p-value & F & p-value & F \\
\hline Age & $\mathbf{0 . 0 0 1}$ & $\mathbf{1 2 . 6 3 3}$ & $\mathbf{0 . 0 0 1}$ & $\mathbf{1 2 . 4 3 3}$ \\
\hline V1 & $\mathbf{0 . 0 3 1}$ & $\mathbf{4 . 7 6 7}$ & $<\mathbf{0 . 0 0 0 1}$ & $\mathbf{1 9 . 2 7 9}$ \\
\hline M1 & $<\mathbf{0 . 0 0 0 1}$ & $\mathbf{3 2 . 2 4 7}$ & $<\mathbf{0 . 0 0 0 1}$ & $\mathbf{1 7 . 0 9 5}$ \\
\hline A1 & 0.602 & 0.511 & 0.775 & 0.256 \\
\hline Group & 0.767 & 0.767 & 0.658 & 0.420 \\
\hline V1 & 0.112 & 2.241 & $\mathbf{0 . 0 4 8}$ & $\mathbf{3 . 1 3 7}$ \\
\hline M1 &
\end{tabular}

Table 5. The effect of age and group on gray matter volume and thickness in the visual (V1), motor (M1), and auditory cortices (A1). Significant results of multivariate analysis of variance are shown in bold. Adjustment for multiple comparisons: Bonferroni. Top: the effect of age on gray matter volume and surface thickness. Bottom: the effect of group on the gray matter volume and surface thickness, with age included as a covariate.

preservation of the lingual gyrus in relation to tinnitus. The precise role of the lingual gyrus in tinnitus remains unclear. Other studies reported that the lingual gyrus is linked to better preserved cognitive function in patients with major depressive disorder ${ }^{49}$, and that larger lingual gyrus volume correlated with better performance on memory tasks ${ }^{50,51}$. In summary, these results suggest a relation between the possible preservation of cognitive functions mediated by the lingual gyrus and the presence of tinnitus. 
Differentiation with other studies on tinnitus. The present study showed that larger gray matter volume in the lingual gyri was specifically related to the presence of tinnitus in individuals with hearing loss. In contrast, previous studies reported tinnitus related reductions in gray matter volume or surface thickness in multiple areas. For instance, differences in the prefrontal cortex ${ }^{32-37,39}$, the supra marginal gyrus ${ }^{33}$, insular $\operatorname{cortex}^{36}$, and cingulate area $^{36}$ were reported. Yet, our study showed that changes in these areas were related to age, and differences in the gray matter of the cingulate area were related to hearing loss. These findings are consistent with the results of Wong et al. ${ }^{27}$. In their study it was reported that a significant relation was present between age, not hearing loss, and surface thickness of the superior frontal gyrus. In addition to the aforementioned areas, other studies reported differences in the subcallosal area ${ }^{35,38}$, and the pre and postcentral gyrus ${ }^{36}$ in relation to tinnitus. However, in our study no significant differences were identified that related to these areas in any of our contrasts. In line with previous reports, the right cluster identified in the lingual gyrus expanded to include the precuneus $^{32}$. To elaborate, if age was omitted as a covariate in our analyses or if we used an uncorrected threshold this resulted in the detection of significant volumetric differences related to hearing loss in the aforementioned areas. Since tinnitus patients often have additional hearing loss and are generally older than control groups, these confounds may explain the uncertainty and contradictory reports on structural differences in tinnitus ${ }^{32,40,52}$. Therefore, some of the results described in earlier studies may reflect (small) differences in hearing loss or age, and are not specifically related to the presence of tinnitus.

Beyond the auditory system: cognition. Our results showed that, regardless of age, several brain areas contained less gray matter in participants with hearing loss, and this effect is more pronounced in hearing loss without tinnitus than in hearing loss with tinnitus. The areas identified in our analyses are in line with areas that are reported to show an aggravated decline of cortical volume $e^{25,26,28}$ and surface thickness ${ }^{30}$ in hearing loss and cognitive impairment. Atrophy of several brain areas beyond the primary auditory system has been related to hearing loss, and this widespread loss of cortical gray matter has the potential to affect a wide range of cortical processes that extend beyond auditory processing. The gray matter areas identified in our study are consistent with those reported in previous longitudinal studies that indicated that in age-related hearing loss the decline in these gray matter areas is progressive over time and relates to cognitive impairments ${ }^{10,28}$.

The nature of the relation between hearing loss and cognitive decline is still debated. It has been suggested that a similar mechanism of progressive loss of brain efficiency can result in both central hearing loss and cognitive decline $^{4,14,53,54}$. Directionality of this effect has been suggested, for example the proposal that a decline in central functioning may contribute to poorer speech understanding and thus that cognition can have a big impact on hearing related processes ${ }^{55}$. Moreover, presumably the declining processing speed is the common cause of both cognitive decline and hearing loss, since the auditory system relies on precise timing and a reduction in temporal fine structure can hinder speech processing. On the other hand it has been described that central hearing disorders, or central auditory dysfunctions, often precede cognitive impairment ${ }^{56}$. The latter finding suggests that central hearing dysfunction can be an early marker of cognitive decline. Based on our results, uncompensated hearing loss without tinnitus is linked to a loss of gray matter in several cortical areas that are implicated in cognitive decline, whereas in the presence of tinnitus these areas are better preserved.

Overall, it is important to note that the differences between participant groups may have developed as a result of hearing loss or tinnitus. Alternatively, differences may have existed before tinnitus or hearing loss developed. For example, it is conceivable that some pre-existing morphological characteristics correspond to a susceptibility to develop hearing loss or tinnitus. Based on our study, we cannot distinguish between gray matter differences that developed over time and pre-existing conditions. An additional factor that may influence the outcomes is that none of our participants had hearing aids to compensate for their hearing loss, or tinnitus maskers to alleviate their tinnitus. Hearing aids and tinnitus maskers may impact the brain by generating more input and hence affect cortical plasticity.

Limitations. The effect of tinnitus on gray matter morphology in this study was derived from the contrast between hearing loss without tinnitus compared to hearing loss with tinnitus. Our results may thus only pertain to tinnitus associated with hearing loss. The possibility exists that tinnitus without the presence of hearing loss affects the gray matter morphology in a different manner.

\section{Conclusion}

This study showed age-related gray matter atrophy in several brain areas, both within and outside of the auditory pathway. With the aid of volumetric and surface-based methods, we showed that age-related hearing loss without tinnitus is related to additional decreases in brain volume of the temporal, frontal, cingular, and parietal areas. The largest differences in gray matter morphometry were caused by age, after which the presence of hearing loss was associated most strongly with a decline in cortical gray matter. Interestingly, the addition of tinnitus in hearing loss individuals appears to reduce the amount of gray matter decline since the gray matter of the hearing loss with tinnitus was not significantly different from that of the control group. The lingual gyrus in particular is larger or better preserved in the presence of tinnitus. These findings confirm that hearing loss has impact beyond the auditory system and is an important factor to consider in the treatment of cognitive impairments.

\section{Methods}

Participants. One hundred and thirteen participants were included in a larger MRI study at the University Medical Center Groningen, the Netherlands. Three people did not complete all aspects of the research, one data file was corrupted in the transfer process, and in an additional nine scans either the contrast between white and grey matter was insufficient for the segmentation algorithm to work reliably, or movement during anatomical 
scanning rendered the data with artefacts. This resulted in complete and sufficient data for a total of 99 participants, of which 39 had tinnitus and hearing loss, 21 participants with hearing loss but without tinnitus, and 39 healthy controls with no tinnitus and no or minimal hearing loss. The study was approved by the Medical Ethical Committee of the University of Groningen, performed in accordance with all relevant regulations and informed consent was obtained from all participants.

Auditory thresholds. The hearing levels of all participants were assessed with pure tone audiometry. Thresholds were determined in a sound attenuating booth for octave frequencies ranging from $0.25 \mathrm{ko} \mathrm{kHz}$, and additionally at 3 and $6 \mathrm{kHz}$. The average hearing loss was quantified as the mean of the hearing thresholds at 2, 4, and $8 \mathrm{kHz}$ of both ears (high-frequency pure-tone average; HF-PTA). A matching procedure was used to estimate tinnitus pitch and loudness. The majority of the participants perceived high frequency tinnitus $(\mathrm{n}=35,>2 \mathrm{kHz})$, and the remaining four participants reported the perception of broadband noise. None of the participants compensated their hearing loss with hearing aids, or ameliorated their tinnitus with maskers. Participants were required to have hearing thresholds better than $40 \mathrm{~dB}$ SPL at $1 \mathrm{kHz}$ to meet the inclusion criteria for a functional task-based scan that was obtained in the same sequence as the anatomical images. This resulted in the inclusion of participants with high frequency hearing loss only.

MRI data acquisition. MRI scanning was performed at the Neuro Imaging Center in Groningen, on a 3.0 T Philips Intera MRI scanner (Best, the Netherlands). A SENSE 32-channel head coil was used to obtain a whole brain T1 weighted anatomical image with a voxel size of $1 \times 1 \times 1 \mathrm{~mm}$ (TR $10.4 \mathrm{~ms}$, TE $5.7 \mathrm{~ms}$, Acquisition matrix $256 \times 200)$.

Data analysis. All T1-images were aligned according to the individual anatomical anterior-posterior commissures in Vistasoft-Master, software run in Matlab2018a. Further analyses were done in CAT12, a structural imaging-oriented SPM toolbox ${ }^{41}$, and included segmentation, normalization and smoothing. Total Intracranial Volume (TIV) was estimated for all participants to correct for differences in head and brain size. After these steps were performed, a sample quality check was run on the segmented images to determine if image quality was sufficiently high; all included images scored above $86 \%$. This score is based on a measure of homogeneity of the sample and is used to determine image quality after pre-processing (i.e. mean correlation measure) and it was run with TIV as a nuisance variable. Data with a low mean correlation (i.e. low image quality) or large differences to the mean score were manually checked for sufficient image quality. If this confirmed the low image quality, images were excluded from further analysis.

The included images were smoothed with an 8-mm Gaussian kernel. The subsequent analyses were performed on the modulated gray matter volume images (VBM) and the surface-based thickness measures (SBM). Modulated gray matter images were scaled by the amount of contraction induced by non-linear spatial normalization. The value of each voxel was multiplied with the Jacobian determinant obtained with spatial normalization. After the pre-processing and first level analysis, second level whole group and group wise analyses were performed (Mechelli et al., 2005).

A multiple regression model was run on the modulated gray matter images with sex, age, the presence of tinnitus, and average hearing loss as covariates to check the association between these covariates and the gray matter volume data. Then, two sample t-tests were performed on both the gray matter volume and surface-based thickness measures to test group differences in a pairwise manner. Threshold Free Cluster Enhancement (TFCE) was used to assess the cluster-wise differences in gray matter volume between the groups ${ }^{57}$. This non-linear method can detect both diffuse low-amplitude signals and sharp focal signals. In order to test for statistical significance, TFCE computes p-values for each voxel via permutation testing.

\section{Data availability}

The dataset analysed in this study is no publicly available due to privacy restrictions. The data can be made available upon reasonable request to the corresponding author.

Received: 23 December 2019; Accepted: 26 November 2020

Published online: 11 December 2020

\section{References}

1. Mathers, C., Smith, A. \& Concha, M. Global burden of hearing loss in the year 2000. in Global Burden of Disease. https://www. who.int/healthinfo/statistics/bod_hearingloss.pdf (2000).

2. OMS. Addressing the Rising Prevalence of Hearing Loss. https://doi.org/10.1080/09687599.2011.589198 (Organización Mundial de la Salud, 2018)

3. Füllgrabe, C., Moore, B. C. J. \& Stone, M. A. Age-group differences in speech identification despite matched audiometrically normal hearing: contributions from auditory temporal processing and cognition. Front. Aging Neurosci. 6, 347 (2014).

4. Humes, L. E., Kidd, G. R. \& Lentz, J. J. Auditory and cognitive factors underlying individual differences in aided speech-understanding among older adults. Front. Syst. Neurosci. 7, 55 (2013).

5. Akeroyd, M. A. Are individual differences in speech reception related to individual differences in cognitive ability? A survey of twenty experimental studies with normal and hearing-impaired adults. Int. J. Audiol. 47, S53-S71 (2008).

6. Houtgast, T. \& Festen, J. M. On the auditory and cognitive functions that may explain an individual's elevation of the speech reception threshold in noise. Int. J. Audiol. 47, 287-295 (2008).

7. Heywood, R. et al. Hearing loss and risk of mild cognitive impairment and dementia: Findings from the Singapore Longitudinal Ageing Study. Dement. Geriatr. Cogn. Disord. 43, 259-268 (2017).

8. Uhlmann, R. F., Larson, E. B., Rees, T. S., Koepsell, T. D. \& Duckert, L. G. Relationship of hearing impairment to dementia and cognitive dysfunction in older adults. JAMA J. Am. Med. Assoc. 261, 1916 (1989). 
9. Lin, F. R. \& Albert, M. Hearing loss and dementia-Who is listening?. Aging Ment. Health 18, 671-673 (2014).

10. Uchida, Y. et al. Age-related hearing loss and cognitive decline - The potential mechanisms linking the two. Auris Nasus Larynx 46, 1-9 (2019).

11. Panza, F., Solfrizzi, V. \& Logroscino, G. Age-related hearing impairment-A risk factor and frailty marker for dementia and AD. Nat. Rev. Neurol. 11, 166-175 (2015).

12. Panza, F. et al. Sensorial frailty: age-related hearing loss and the risk of cognitive impairment and dementia in later life. Ther. Adv. Chronic Dis. https://doi.org/10.1177/2040622318811000 (2018).

13. Livingston, G. et al. Dementia prevention, intervention, and care. Lancet (London, England) 390, 2673-2734 (2017).

14. Jafari, Z., Kolb, B. E. \& Mohajerani, M. H. Age-related hearing loss and tinnitus, dementia risk, and auditory amplification outcomes. Ageing Res. Rev. 56, 100963 (2019).

15. Robertson, D. \& Irvine, D. R. F. Plasticity of frequency organization in auditory cortex of guinea pigs with partial unilateral deafness. J. Comp. Neurol. 282, 456-471 (1989).

16. Mühlnickel, W., Elbert, T., Taub, E. \& Flor, H. Reorganization of auditory cortex in tinnitus. Proc. Natl. Acad. Sci. U. S. A. 95, 10340-10343 (1998).

17. Rauschecker, J. P. Auditory cortical plasticity: A comparison with other sensory systems. Trends Neurosci. 22, 74-80 (1999).

18. Eggermont, J. J. \& Roberts, L. E. The neuroscience of tinnitus. Trends Neurosci. 27, 676-682 (2004).

19. Norena, A. J. \& Eggermont, J. J. Enriched acoustic environment after noise trauma reduces hearing loss and prevents cortical map reorganization. J. Neurosci. 25, 699-705 (2005).

20. Eggermont, J. J. Cortical tonotopic map reorganization and its implications for treatment of tinnitus. Acta Otolaryngol. Suppl. 9-12 https://doi.org/10.1080/03655230600895259 (2006).

21. Wang, Y. et al. The characteristics of cognitive impairment in subjective chronic tinnitus. Brain Behav. 8, e00918 (2018).

22. Tegg-Quinn, S., Bennett, R. J., Eikelboom, R. H. \& Baguley, D. M. The impact of tinnitus upon cognition in adults: A systematic review. Int. J. Audiol. 55, 533-540 (2016).

23. Wang, X. et al. Alterations in gray matter volume due to unilateral hearing loss. Sci. Rep. 6, 25811 (2016).

24. Husain, F. T. et al. Neuroanatomical changes due to hearing loss and chronic tinnitus: A combined VBM and DTI study. Brain Res. 1369, 74-88 (2011).

25. Peelle, J. E., Troiani, V., Grossman, M. \& Wingfield, A. Hearing loss in older adults affects neural systems supporting speech comprehension. J. Neurosci. 31, 12638-12643 (2011).

26. Eckert, M. A. et al. Auditory cortex signs of age-related hearing loss. J. Assoc. Res. Otolaryngol. 13, 703-713 (2012),

27. Wong, P. C. M., Ettlinger, M., Sheppard, J. P., Gunasekera, G. M. \& Dhar, S. Neuroanatomical characteristics and speech perception in noise in older adults. Ear Hear. 31, 471-479 (2010).

28. Lin, F. R. et al. Association of hearing impairment with brain volume changes in older adults. Neuroimage 90, 84-92 (2014).

29. Profant, O. et al. Diffusion tensor imaging and MR morphometry of the central auditory pathway and auditory cortex in aging. Neuroscience. https://doi.org/10.1016/j.neuroscience.2013.12.010 (2014).

30. Ren, F. et al. Gray matter atrophy is associated with cognitive impairment in patients with presbycusis: A comprehensive morphometric study. Front. Neurosci. 12, 744 (2018).

31. Alfandari, D. et al. Brain volume differences associated with hearing impairment in adults. Trends Hear. 22, 2331216518763689 (2018).

32. Allan, T. W. et al. Neuroanatomical alterations in tinnitus assessed with magnetic resonance imaging. Front. Aging Neurosci. 8, 221 (2016).

33. Leaver, A. M. et al. Cortico-limbic morphology separates tinnitus from tinnitus distress. Front. Syst. Neurosci. 6, 21 (2012).

34. Aldhafeeri, F. M., MacKenzie, I., Kay, T., Alghamdi, J. \& Sluming, V. Neuroanatomical correlates of tinnitus revealed by cortical thickness analysis and diffusion tensor imaging. Neuroradiology 54, 883-892 (2012).

35. Leaver, A. M. et al. Dysregulation of limbic and auditory networks in tinnitus. Neuron 69, 33-43 (2011).

36. Schecklmann, M. et al. Cluster analysis for identifying sub-types of tinnitus: A positron emission tomography and voxel-based morphometry study. Brain Res. 1485, 3-9 (2012).

37. Boyen, K., Langers, D. R. M., de Kleine, E. \& van Dijk, P. Gray matter in the brain: Differences associated with tinnitus and hearing loss. Hear. Res. 295, 67-78 (2013).

38. Muhlau, M. et al. Structural brain changes in tinnitus. Cereb. Cortex 16, 1283-1288 (2006).

39. Mahoney, C. J. et al. Structural neuroanatomy of tinnitus and hyperacusis in semantic dementia. J. Neurol. Neurosurg. Psychiatry 82, 1274-1278 (2011).

40. Vanneste, S., Van De Heyning, P. \& De Ridder, D. Tinnitus: a large VBM-EEG correlational study. PLoS ONE 10, e0115122 (2015).

41. Dahnke, R., Yotter, R. A. \& Gaser, C. Cortical thickness and central surface estimation. Neuroimage 65, 336-348 (2013).

42. Glasser, M. F. et al. A multi-modal parcellation of human cerebral cortex. Nature 536, 171-178 (2016).

43. Good, C. D. et al. A voxel-based morphometric study of ageing in 465 normal adult human brains. Neuroimage 14, 21-36 (2001).

44. Resnick, S. M., Pham, D. L., Kraut, M. A., Zonderman, A. B. \& Davatzikos, C. Longitudinal magnetic resonance imaging studies of older adults: A shrinking brain. J. Neurosci. 23, 3295-3301 (2003).

45. Hutton, C., Draganski, B., Ashburner, J. \& Weiskopf, N. A comparison between voxel-based cortical thickness and voxel-based morphometry in normal aging. Neuroimage 48, 371-380 (2009).

46. Hinkley, L. B., Mizuiri, D., Hong, O., Nagarajan, S. S. \& Cheung, S. W. Increased striatal functional connectivity with auditory cortex in tinnitus. Front. Hum. Neurosci. 9, 568 (2015).

47. Schmidt, S. A., Akrofi, K., Carpenter-Thompson, J. R. \& Husain, F. T. Default mode, dorsal attention and auditory resting state networks exhibit differential functional connectivity in tinnitus and hearing loss. PLoS ONE 8, e76488 (2013).

48. Husain, F. T., Akrofi, K., Carpenter-Thompson, J. R. \& Schmidt, S. A. Alterations to the attention system in adults with tinnitus are modality specific. Brain Res. 1620, 81-97 (2015).

49. Jung, J. et al. Impact of lingual gyrus volume on antidepressant response and neurocognitive functions in major depressive disorder: A voxel-based morphometry study. J. Affect. Disord. 169, 179-187 (2014).

50. Walhovd, K. B. et al. Regional cortical thickness matters in recall after months more than minutes. Neuroimage 31, 1343-1351 (2006).

51. Kalpouzos, G. et al. Voxel-based mapping of brain gray matter volume and glucose metabolism profiles in normal aging. Neurobiol. Aging 30, 112-124 (2009).

52. Shore, S. E., Roberts, L. E. \& Langguth, B. Maladaptive plasticity in tinnitus - Triggers, mechanisms and treatment. Nat. Rev. Neurol. 12, 150-160 (2016).

53. Humes, L. E., Busey, T. A., Craig, J. \& Kewley-Port, D. Are age-related changes in cognitive function driven by age-related changes in sensory processing?. Atten. Percept. Psychophys. 75, 508-524 (2013).

54. Sardone, R. et al. The age-related central auditory processing disorder: Silent impairment of the cognitive ear. Front. Neurosci. 13, 619 (2019).

55. Moore, D. R. et al. Relation between speech-in-noise threshold, hearing loss and cognition from 40-69 years of age. PLoS ONE 9 , e107720 (2014).

56. Gates, G. A., Beiser, A., Rees, T. S., D’Agostino, R. B. \& Wolf, P. A. Central auditory dysfunction may precede the onset of clinical dementia in people with probable Alzheimer's disease. J. Am. Geriatr. Soc. 50, 482-488 (2002). 
57. Smith, S. M. \& Nichols, T. E. Threshold-free cluster enhancement: Addressing problems of smoothing, threshold dependence and localisation in cluster inference. Neuroimage 44, 83-98 (2008).

\section{Acknowledgements}

This study was supported by the American Tinnitus Foundation, Dorhout Mees Foundation, Heinsius-Houbolt Foundation, NWO, Stichting Steunfonds Audiologie and the William Demant Foundation.

\section{Author contributions}

P.D. and E.A.K. designed the project. E.A.K. performed the experiment, and analysed the data. P.D. and E.K. aided in interpreting the results. P.D. supervised the work. E.A.K. wrote the manuscript with support and input from P.D. and E.K.

\section{Competing interests}

The authors declare no competing interests.

\section{Additional information}

Correspondence and requests for materials should be addressed to E.A.K.

Reprints and permissions information is available at www.nature.com/reprints.

Publisher's note Springer Nature remains neutral with regard to jurisdictional claims in published maps and institutional affiliations.

(c) (1) Open Access This article is licensed under a Creative Commons Attribution 4.0 International License, which permits use, sharing, adaptation, distribution and reproduction in any medium or format, as long as you give appropriate credit to the original author(s) and the source, provide a link to the Creative Commons licence, and indicate if changes were made. The images or other third party material in this article are included in the article's Creative Commons licence, unless indicated otherwise in a credit line to the material. If material is not included in the article's Creative Commons licence and your intended use is not permitted by statutory regulation or exceeds the permitted use, you will need to obtain permission directly from the copyright holder. To view a copy of this licence, visit http://creativecommons.org/licenses/by/4.0/.

(c) The Author(s) 2020 